\title{
ON THE CHARACTERISTIC HOMOMORPHISM OF A DISCRETE UNIFORM SUBGROUP OF A NILPOTENT LIE GROUP
}

\author{
BY FRANZ KAMBER ${ }^{1}$
}

Communicated by N. E. Steenrod, January 17, 1968

1. In [2] A. Grothendieck quotes an example, due to J. P. Serre, of a complex algebraic nilpotent Lie group $G$ and a discrete uniform subgroup $\alpha: \Gamma \subset G$, such that the characteristic homomorphism induced in the rational cohomology of the classifying spaces

$$
B \alpha^{*}: H^{*}\left(B_{G}, \boldsymbol{Q}\right) \rightarrow H^{*}\left(B_{\Gamma}, \boldsymbol{Q}\right) \cong H^{*}(\Gamma, \boldsymbol{Q})
$$

is not trivial. The purpose of this note is to generalize this example to nilpotent Lie groups admitting a discrete uniform subgroup.

Let $G^{\prime}$ be a simply connected and connected nilpotent (real) Lie group of dimension $n$ with center $Z\left(G^{\prime}\right)$ and $\alpha^{\prime}: \Gamma^{\prime} \subset G^{\prime}$ a discrete uniform subgroup of $G^{\prime}$ (i.e., $G^{\prime} / \Gamma^{\prime}$ compact). Then $A=\Gamma^{\prime} \cap Z\left(G^{\prime}\right)$ is a free abelian group of rank $m=\operatorname{dim} Z\left(G^{\prime}\right)>0$. Consider $G=G^{\prime} / A$ and $\Gamma=\Gamma^{\prime} / A$ with the natural inclusion $\alpha: \Gamma \subset G$ and the induced map in classifying spaces $B \alpha: B_{\Gamma} \rightarrow B_{G}$. It is clear that $\Gamma$ is a discrete uniform subgroup of $G$.

THEOREM 1.1. The rational characteristic homomorphism

$$
B \alpha^{*}: H^{*}\left(B_{G}, Q\right) \rightarrow H^{*}\left(B_{\Gamma}, Q\right)=H^{*}(\Gamma, Q)
$$

is trivial (i.e. zero in positive dimensions) if and only if $G^{\prime}$ is the vector group $R^{n}$.

Since $G^{\prime}$ is contractible, $G=G^{\prime} / A$ is a space of type $K(A, 1)$ and hence the cohomology of its classifying space is given by $H^{*}\left(B_{G}, Z\right)$ $=\boldsymbol{Z}\left[x_{1}, \cdots, x_{m}\right], \operatorname{deg}\left(x_{k}\right)=2$. Moreover $B \alpha$ is the classifying map of the flat $G$-bundle $\eta: E_{\Gamma} \times_{\Gamma} G \rightarrow B_{\Gamma}$, where $E_{\Gamma}$ is the total space of the universal $\Gamma$-bundle [4], [5]. The classes $x_{k}(\eta)=B \alpha^{*}\left(x_{k}\right) \in H^{2}(\Gamma, Z)$ are then the characteristic classes over $\boldsymbol{Z}$ of the $G$-bundle $\eta$.

Corollary 1.2. The integral characteristic classes $x_{k}(\eta), k=1$, $\cdots, m$ of the flat $G$-bundle $\eta$ are torsion classes if and only if $G^{\prime}$ is the vector group $R^{n}$.

${ }^{1}$ The author was supported by a National Science Foundation grant at The Institute for Advanced Study, Princeton. 
Since $B_{\Gamma}$ can be realized as a compact manifold ( $\left.\$ 4\right)$, it has the homotopy type of a finite $\mathrm{CW}$-complex; hence 1.2 is just a reformulation of 1.1 .

Theorem 1.1 is in sharp contrast to [4, Theorems $2.2,3.4,3.5]$ and [2, Theorem 7.1] where sufficient conditions for the triviality of the rational characteristic homomorphism were given and it increases the number of examples $[8],[2,7.5]$ of flat principal bundles with nontrivial rational characteristic classes.

The proof of Theorem 1.1 will be given in $\$ \S 2$ and 3. In $\$ 4$ we will consider a particular realization of $B_{\Gamma}$ as a compact manifold and examine the question whether the tangent bundle of this manifold is associated to the bundle $\eta$ in Corollary 1.2.

I want to thank A. Borel, J. P. Brezin and J. P. Serre for a useful discussion.

2. Proof of Theorem 1.1. The "if" part of the theorem is trivial. In fact, $G^{\prime}=R^{n}$ implies $\Gamma=\{1\}$. The "only if" part will be proved in the formulation of Corollary 1.2. The characteristic classes $x_{k}(\eta)$ $=B \alpha^{*}\left(x_{k}\right) \in H^{2}(\Gamma, \boldsymbol{Z})$ can be given various interpretations which we list in the following

Lemma 2.1 [5, Proposition 4.17], [10, p. 189]. The following elements of $H^{2}(\Gamma, A), A \cong \pi_{1}(G) \cong Z^{m}$ are equal up to sign:

(i) $x(\eta)=\left(x_{k}(\eta)\right)_{k=1, \cdots, m}$.

(ii) $\tau_{\eta}(\iota)$, where $\iota$ is the fundamental class in $H^{1}\left(G, \pi_{1}(G)\right.$ ) $\cong \operatorname{Hom}\left(\pi_{1}(G), \pi_{1}(G)\right)$ corresponding to the identity homomorphism and $\tau_{\eta}$ is the transgression in the bundle $\eta$.

(iii) $\mathfrak{D}(\eta)$, the primary obstruction to a cross section in $\eta$.

(iv) $\delta(\alpha)$, where $\delta$ is the coboundary in the non-abelian cohomology sequence

$$
* \rightarrow H^{1}(\Gamma, A) \rightarrow H^{1}\left(\Gamma, G^{\prime}\right) \rightarrow H^{1}(\Gamma, G) \stackrel{\delta}{\rightarrow} H^{2}(\Gamma, A)
$$

associated to the universal covering sequence

$$
0 \rightarrow A \rightarrow G^{\prime} \rightarrow G \rightarrow 1
$$

of $G$.

(v) $\phi(E)$, the characteristic cohomology class of the central extension

$$
E: 0 \rightarrow A \underset{i}{\rightarrow} \Gamma^{\prime} \underset{q}{\rightarrow} \Gamma \rightarrow 1 .
$$

REMARK 2.2. Lemma 2.1 only makes sense for specific isomorphisms $H^{*}\left(B_{G}, \boldsymbol{Z}\right) \cong Z\left[x_{1}, \cdots, x_{m}\right]$ and $\pi_{1}(G) \cong Z^{m}$. These isomorphisms will be exhibited at the end of $\$ 3$. 
By Lemma 2.1 it is sufficient to show that $G^{\prime}$ is the vector group $R^{n}$ if the class $\phi(E)$ is a torsion class. The spectral sequence of the extension $E$ determines a five-term exact sequence [3, Theorem 4.2]

$$
\begin{aligned}
0 & \rightarrow H^{1}(\Gamma, A) \rightarrow H^{1}\left(\Gamma^{\prime}, A\right) \\
& \overrightarrow{i^{*}} H^{1}(A, A) \underset{\tau}{\rightarrow} H^{2}(\Gamma, A) \underset{q^{*}}{\rightarrow} H^{2}\left(\Gamma^{\prime}, A\right)
\end{aligned}
$$

and it is well known that $\phi(E)=\tau\left(\operatorname{id}_{A}\right)$. If $\phi(E)$ is a torsion class it follows from the exactness of (2.3) that $\phi \circ i=\lambda \cdot \mathrm{id}_{A}$ for some $\lambda \in Z^{+}$ and $\phi \in H^{1}\left(\Gamma^{\prime}, A\right)=\operatorname{Hom}\left(\Gamma^{\prime}, A\right)$ ( $A$ is a trivial $\Gamma^{\prime}$-module).

REMARK 2.4. Up to now our considerations apply equally well to the primary obstruction of flat bundles induced by any homomorphism $\alpha: \Gamma \rightarrow G$ of a discrete group $\Gamma$ into a path-connected topological group $G$. The next lemma, however, will make use of the fact that $G$ is a nilpotent Lie group.

Lemma 2.5. Let $\alpha^{\prime}: \Gamma^{\prime} \rightarrow G^{\prime}$ and $A=\Gamma^{\prime} \cap Z\left(G^{\prime}\right)$, as in $\S 1$. If there exists $a$ homomorphism $\phi \in \operatorname{Hom}\left(\Gamma^{\prime}, A\right)$ such that $\phi \circ i=\lambda \cdot \operatorname{id}_{A}, \lambda \in Z^{+}$where $i: A \rightarrow \Gamma^{\prime}$ is the inclusion, then $G^{\prime}$ is isomorphic to the vector group $R^{n}$.

It is clear from what has already been said that Lemma 2.5 will complete the proof of Theorem 1.1.

3. First we list some known facts about 1-connected and connected nilpotent Lie groups and their discrete uniform subgroups. Let $\alpha^{\prime}: \Gamma^{\prime} \subset G^{\prime}$ be such a pair and $g$ the Lie algebra of $G^{\prime}$. Then there exists a base $\xi_{1}, \cdots, \xi_{n}$ of $g$ such that the map $\rho: \mathfrak{g} \rightarrow G^{\prime}$ defined by $\xi$ $=\sum_{i=1}^{n} \lambda_{i} \cdot \xi_{i m \rho \rho}(\xi)=\prod_{i=1}^{n} \exp \left(\lambda_{i} \cdot \xi_{i}\right)$ is a homeomorphism and $\rho(\xi) \in \Gamma^{\prime}$ if and only if $\lambda_{i} \in \boldsymbol{Z}$ (Malcev coordinates [6]). Moreover the center $\mathfrak{z}(\mathfrak{g})$ of $\mathfrak{g}$ is nontrivial, say of dimension $m>0[1 ; 4$, Corollary 1$]$ and exp: $z(\mathfrak{g}) \rightarrow Z\left(G^{\prime}\right)$ is a homeomorphism [7, Lemma 3]. Hence $Z\left(G^{\prime}\right)$ is a vector group $R^{m}$. We quote the following results as a lemma.

LEMMA 3.1. (i) [6] $G^{\prime}$ has a discrete uniform subgroup if and only if there exists a (nilpotent) Lie algebra $\mathfrak{h}$ over the rationals $Q$ such that $\mathfrak{g}=\mathfrak{h} \otimes Q R$ (i.e. $\mathfrak{g}$ is rational).

(ii) [9, Lemma 2.1] Let $H \subset G^{\prime}$ be a closed connected subgroup, $\Gamma^{\prime} \subset G^{\prime}$ a discrete uniform subgroup. Then $\Gamma^{\prime} \cap H \subset H$ is uniform if the Lie algebra $\mathfrak{h}$ of $H$ is rational.

Since $Z\left(G^{\prime}\right)$ is trivially rational, it follows that $A=\Gamma^{\prime} \cap Z\left(G^{\prime}\right)$ is uniform in $Z\left(G^{\prime}\right)$. Hence $A$ is free abelian of rank $m$ and $Z\left(G^{\prime}\right) / A$ is a torus $T^{m}$.

We are now ready to prove Lemma 2.5 . Consider the diagram 
I968] CHARACTERISTICHOMOMORPHISMOF A DISCRETE UNIFORM SUBGROUP 693

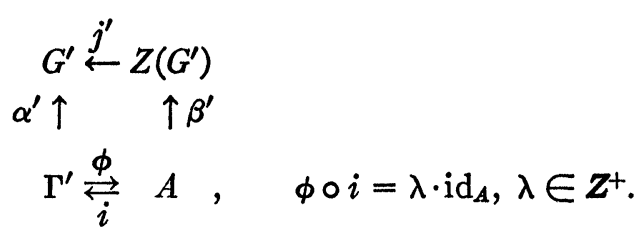

Using Malcev coordinates we define an analytic homomorphism $\Phi: G^{\prime} \rightarrow Z\left(G^{\prime}\right)$ satisfying $\beta^{\prime} \circ \phi=\Phi \circ \alpha^{\prime}$ and $\Phi \circ j^{\prime}=\lambda \cdot \operatorname{id}_{z^{\prime}}$ by $\Phi(g)$ $=\sum_{i=1}^{n} t_{i} \cdot d_{i}$, where $g=\prod_{i=1}^{n} \exp \left(t_{i} \cdot \xi_{i}\right)$ and $d_{i}=\phi\left(\exp \left(\xi_{i}\right)\right.$ ) (we use additive notation in $Z\left(G^{\prime}\right) \cong R^{m}$ and omit $\alpha^{\prime}$ and $\beta^{\prime}$ from the formulae). $\Phi$ extends $\phi$ : In fact, for $\gamma=\prod_{i=1}^{n} \exp \left(t_{i} \cdot \xi_{i}\right) \in \Gamma^{\prime}, t_{i} \in Z$, we have $\Phi(\gamma)=\sum_{i=1}^{n} t_{i} \cdot d_{i}=\sum_{i=1}^{n} t_{i} \cdot \phi\left(\exp \left(\xi_{i}\right)\right)=\phi(\gamma)$ since $\phi$ is a homomorphism. Let $h=\prod_{i=1}^{n} \exp \left(s_{i} \cdot \xi_{i}\right)$; then we have $g h=\prod_{i=1}^{n} \exp \left(u_{i} \cdot \xi_{i}\right)$, $u_{i}=t_{i}+s_{i}+q_{i}\left(t_{1}, \cdots, t_{i-1} ; s_{1}, \cdots, s_{i-1}\right)$ where the $q_{i}$ are polynomials with rational coefficients and integral values for $t_{i}, s_{i} \in \boldsymbol{Z}$ [6]. It follows that $\Phi(g h)=\Phi(g)+\Phi(h)+\Sigma, \Sigma=\sum_{i=1}^{n} q_{i}\left(t_{1}, \cdots ; s_{1}, \cdots\right) \cdot d_{i}$. Since $\phi$ is a homomorphism, the polynomial function $\Sigma$ with values in $Z\left(G^{\prime}\right) \cong R^{m}$ vanishes for $t_{i}, s_{i} \in \boldsymbol{Z}$. Hence it vanishes identically and $\Phi$ is a homomorphism. Finally, $\Phi \circ j^{\prime}: Z\left(G^{\prime}\right) \rightarrow Z\left(G^{\prime}\right)$ extends $\phi \circ i$ $=\lambda \cdot \mathrm{id}_{A}$ and since $A \subset Z\left(G^{\prime}\right)$ is discrete uniform, this extension is unique and given by $\lambda \cdot \mathrm{id}_{Z^{\prime}}$.

Since $Z\left(G^{\prime}\right)$ is a vector group we can define a new homomorphism $\Psi(g)=1 / \lambda \cdot \Phi(g), g \in G^{\prime}$ which now satisfies $\Psi \circ j=\mathrm{id}_{z^{\prime}}$. Hence we have $G^{\prime}=Z\left(G^{\prime}\right) \times G^{\prime \prime}$ with $G^{\prime \prime}=\operatorname{ker}(\Psi)$. $G^{\prime \prime}$ is with $G^{\prime} 1$-connected, connected and nilpotent. Moreover $G^{\prime \prime}$ is centerless and hence trivial by $\left[1 ; 4\right.$, Corollary1]. Therefore $n=m$ and $G^{\prime}=Z\left(G^{\prime}\right)=R^{n}$.

Lemma 3.2. (i) $j: T^{m}=Z\left(G^{\prime}\right) / A \subset G=G^{\prime} / A$ is a maximal compact subgroup of $G, A=\Gamma^{\prime} \cap Z\left(G^{\prime}\right)$.

(ii) $\bar{\alpha}: \Gamma=\Gamma^{\prime} / A \subset G^{\prime} / Z\left(G^{\prime}\right)$ is a discrete uniform subgroup and we have a principal fibration

$$
T^{m} \rightarrow G^{\prime} / \Gamma^{\prime} \rightarrow\left(G^{\prime} / Z\left(G^{\prime}\right)\right) / \Gamma .
$$

Proof. (i) The fibration $Z\left(G^{\prime}\right) / A \rightarrow G^{\prime} / A \rightarrow G^{\prime} / Z\left(G^{\prime}\right)$ shows at once that $Z\left(G^{\prime}\right) / A$ is maximal compact in $G^{\prime} / A$, since $G^{\prime} / Z\left(G^{\prime}\right)$ is contractible. By Lemma 3.1 (ii), $Z\left(G^{\prime}\right) \cdot \Gamma^{\prime}$ is closed in $G^{\prime}$ and hence $\Gamma$ is discrete in $G^{\prime} / Z\left(G^{\prime}\right)$. The fact that (3.3) is a fibration follows now easily. Since $G^{\prime} / \Gamma^{\prime}$ is compact, the same must be true for $\left(G^{\prime} / Z\left(G^{\prime}\right)\right) / \Gamma$.

REMARK 3.3. By Lemma 3.2, $j: T^{m} \subset G$ is a homotopy equivalence and so is the map in classifying spaces, $B j: B_{T^{m} \rightarrow B_{G}}$. The isomorphisms referred to in Remark 2.2 are those induced by $j$ resp. $B j$ (we choose standard generators in $T^{m}$ ).

4. Let $G$ be a connected Lie group, $j: K \subset G$ a maximal compact subgroup, $\alpha: \Gamma \subset G$ a discrete uniform subgroup and assume for sim- 
plicity that $\Gamma \cap Z(G)=0$ and that $\Gamma$ is torsion-free. Then $\Gamma$ acts freely and properly discontinuous on $K \backslash G$, which is topologically a euclidean space; hence the compact manifold $(K \backslash G) / \Gamma$ is of type $K(\Gamma, 1)$ and can be taken as a classifying space $B_{\Gamma}$. With respect to the parallelization of $G$ by right invariant vector fields the tangent bundle of $B_{\Gamma}$ is of the form $\left(\left(\mathfrak{g} / \mathfrak{l} \times{ }_{K} G\right) / \Gamma \rightarrow(K \backslash G) / \Gamma\right.$, where $K$ acts on $\mathfrak{g} / \mathfrak{l}$ by the isotropy representation $\rho: K \rightarrow \mathrm{GL}^{+}(\mathfrak{g} / \mathfrak{l})$. It is then easily seen that the composite homomorphism

(4.1) $H^{*}\left(B_{\mathrm{GL}^{+}}, \mathrm{Q}\right) \stackrel{B \rho^{*}}{\longrightarrow} H^{*}\left(B_{K}, Q\right) \stackrel{\left(B j^{*}\right)^{-1}}{\cong} H^{*}\left(B_{G}, Q\right) \stackrel{B \alpha^{*}}{\longrightarrow} H^{*}\left(B_{\Gamma}, Q\right)$

is the characteristic homomorphism of the tangent bundle of $B_{\mathrm{r}}$, so that this tangent bundle is in a weak sense associated to the flat $G$-bundle $\eta: E_{\Gamma} \times_{\Gamma} G \rightarrow B_{\Gamma}, E_{\Gamma}=K \backslash G$ with classifying $\operatorname{map} B \alpha: B_{\Gamma} \rightarrow B_{G}$.

Let now $\Gamma$ and $G$ as in Theorem 1.1. Then by Lemma $3.2 K=T^{m}$ and $B_{\Gamma}=\left(G^{\prime} / Z\left(G^{\prime}\right)\right) / \Gamma=\left(G / T^{m}\right) / \Gamma$. Since $T^{m}$ is in the center of $G$, it follows that the isotropy representation $\rho$ is trivial and that the tangent bundle of $B_{\Gamma}$ is trivial. Looking at (4.1) one sees that the characteristic classes $x_{k}(\eta)=B \alpha^{*}\left(x_{k}\right), k=1, \cdots, m$ of the flat $G$-bundle $\eta$ in Corollary 1.2 are not tangent classes of $B_{\mathrm{r}}$.

A detailed study of the relation between the characteristic classes of the representation $\alpha: \Gamma \subset G$ and the tangent classes of the manifold $B_{\Gamma}=(K \backslash G) / \Gamma$ in the more general case described above will be made in a subsequent paper.

\section{REFERENCES}

1. N. Bourbaki, Groupes et algebres de Lie, Hermann, Paris, 1960.

2. A. Grothendieck, Classes de Chern et représentations linéaires des groupes discrets, preprint.

3. G. Hochschild and J. P. Serre, Cohomology of group extensions, Trans. Amer. Math. Soc. 74 (1953), 110-134.

4. F. Kamber and $\mathrm{Ph}$. Tondeur, The characteristic homomorphism of flat bundles, Topology 6 (1967), 153-159.

5. - Flat bundles and characteristic classes of group representations, Amer. J. Math. 89 (1967), 857-886.

6. A. I. Malcev, On a class of homogeneous spaces, Izv, Akad. Nauk SSSR Ser. Mat. 13 (1949), 9-32; Amer. Math. Soc. Trans. No. 39 (1949).

7. Y. Matsushima, On the discrete subgroups and homogeneous spaces of nilpotent Lie groups, Nagoya Math. J. 2 (1951), 95-110.

8. J. Milnor, On the existence of a connection with curvature zero, Comment. Math. Helv. 32 (1957), 215-223.

9. C. C. Moore, Decomposition of unitary representations defined by discrete suhgroups of nilpotent Lie groups, Ann. Math. 82 (1965), 146-182.

10. N. Steenrod, The topology of fiber bundles, Princeton Univ. Press, Princeton, N. J., 1951.

The Institute for Advanced Study 